C2020. Licensed under the Creative Commons Attribution-NonCommercial-NoDerivatives 4.0 International http://creativecommons.org/about/downloads

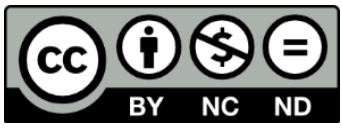

DOI of final article - https://dx.doi.org/10.1016/j.actaastro.2020.03.046 


\title{
Dynamics and Combustion of Single Aluminium Agglomerate in Solid Propellant Environment
}

\author{
V.N. Emelyanov ${ }^{1}$, I.V. Teterina ${ }^{1}$, K.N. Volkov ${ }^{2}$ \\ ${ }^{1}$ Baltic State Technical University, St Petersburg, 190005, Russia \\ ${ }^{2}$ Kingston University, London, SW15 3DW, United Kingdom
}

\begin{abstract}
Aluminized composite propellant used in solid rocket motors contain a lot of aluminium particles because high combustion energy is generated and propulsion efficiency increases by burning aluminium particles. The combustion of aluminum occurs in a significant portion of the combustion chamber and produces aluminum oxide smokes and residues that are carried into the flowfield. Agglomerates have non-spherical shape, and consist of aluminium droplet and oxide particle (oxide cap) attached to the droplet. Unlike the liquid droplet ignition, the solid oxide film blocks the liquid aluminum from the penetration of the oxidizer hence prevents the particle from its ignition. Development of robust models of aluminum particle dynamics is essential in the design of advanced propulsion systems. The mathematical model of two-phase flow around a single aluminum droplet with oxide cap is developed. The model solves the continuity, momentum, energy and species continuity equations simultaneously to obtain the species and temperature profiles and the burning time of droplet. The results of numerical simulations are compared with predictions from semi-empirical correlations and computational data.
\end{abstract}

\section{Keywords}

Flight safety; Solid rocket motor; Agglomeration; Droplet; Oxide cap; Two-phase flow; Combustion; Burning time

\section{Nomenclature}

Latin symbols

$\begin{array}{ll}C & \text { Mass fraction } \\ c_{p} & \text { Specific heat capacity at constant pressure } \\ D & \text { Diameter of droplet } \\ e & \text { Total energy per unit mass } \\ F_{x}, F_{y}, F_{z} & \text { Fluxes } \\ G & \text { Diffusive flux } \\ h & \text { Specific enthalpy } \\ J & \text { gas phase rate } \\ k & \text { Reaction constant } \\ m & \text { Mass flow rate } \\ M & \text { Molar mass }\end{array}$

Corresponding author: k.volkov@kingston.ac.uk 


$\begin{array}{ll}n & \text { Number of particles per unit volume } \\ p & \text { Pressure } \\ q & \text { Heat flux } \\ Q & \text { Vector of conservative variables } \\ R & \text { Radius of droplet } \\ S & \text { Source term } \\ \operatorname{Re} & \text { Reynolds number } \\ s & \text { Mass consumption rate } \\ \text { Sc } & \text { Schmidt number } \\ t & \text { Time } \\ T & \text { Temperature } \\ u & \text { Rate of progress variable } \\ \boldsymbol{v} & \text { Velocity vector } \\ v_{x}, v_{y}, v_{z} & \text { Cartesian velocity components } \\ V & \text { Volume } \\ \mathrm{We} & \text { Weber number } \\ x, y, z & \text { Cartesian coordinates } \\ Y & \text { Mass fraction }\end{array}$

Greek symbols

$\beta$

$\gamma$

$\theta$

$\lambda$

$\mu$

$\nu$

\section{$\rho$}

$\sigma$

\section{$\tau$}

$\chi$

$\omega$

Subscripts

$\begin{array}{ll}b & \text { Burning } \\ f & \text { Forward } \\ F & \text { Fuel } \\ g & \text { Gas phase } \\ n & \text { Normal } \\ O & \text { Oxidizer } \\ p & \text { Particle } \\ r & \text { Reversed } \\ s & \text { Surface } \\ w & \text { Free surface of droplet } \\ \tau & \text { Tangential } \\ 0 & \text { Initial } \\ * & \text { Critical } \\ \infty & \text { Infinity }\end{array}$

Specific heat ratio

Contact angle

Thermal conductivity

Dynamic viscosity

Kinematic viscosity

Density

Surface tension

Stress tensor

Stoichiometric coefficient

Production rate of specie

Surface area blocked by oxide cap 


\section{Introduction}

Aluminized composite propellants used in solid rocket motors (SRMs) contain a lot of metal (usually aluminium) particles because high combustion energy is generated and propulsion efficiency increases by burning metal particles $[1,2]$. Aluminized propellants typically contain close to $20 \%$ aluminum by weight. Nominal particle sizes are typically between 20 and 30 $\mu \mathrm{m}$. Incomplete aluminum combustion inside the motor decreases the thrust and creates twophase flow losses [3]. Rocket systems that use aluminized composite propellants have failed to reach the full potential predicted from the metal combustion, due to performance losses introduced with the two-phase particulate flow of the molten aluminum particles and smoke oxide particles. Another problem with aluminum in SRMs is slag formation. Slag is formed when molten alumina gets trapped in the aft-dome region around the submerged nozzle. Slag affects performance by adding to the weight of the motor causing excessive heating and subsequent insulation erosion, going down flight dynamics and flight safety. The use of aluminum particles for acoustic instability suppression is highly sensitive to particle size, and the control of agglomeration sizes becomes an essential factor in SRM design [4]. Detailed knowledge of condensed phase characteristics as residue size and burning time are essential to improve SRM performance and reliability.

Composite solid propellants consist of a multi-phase mixture of oxidizing and metal powders bound together by a polymer matrix, generating a heterogeneous material. During the combustion, oxidizer and binder a re-subjected to thermal decomposition with release of oxidizing and combustion products. A number of different analytical models have been proposed to determine the aluminum agglomeration process and the aluminum size in composite propellants. Many agglomeration models are based on the pocket concept [5-7]. The main idea behind the pocket model is that aluminum particles gather in the pockets between coarse oxidizer particles during the propellant mixing process. The resulting proximity of aluminum particles inside pockets causes them to form agglomerates during the combustion. All aluminum particles inside model-defined pockets are assumed to combine and form an agglomerate [8]. Agglomeration also involves neighboring pockets (inter-pocket process in slow-burning propellants), as well as coming to pocket break-up if residence time of particles on surface is small enough (sub-pocket process for higher regression rates).

When an aluminum particle reaches the burning surface, ignition is delayed by the fuelrich environment and the oxide shell around the particle. Expansion of the aluminum particle center due to melting causes the oxide shell to crack exposing aluminum to oxidizing species. When ignition occurs, the aluminum particle or agglomerate balls up into a sphere and leaves the surface [3]. Combustion of aluminum particles produces aluminum oxide smokes and residues that are carried into the flowfield. A distinguish feature of aluminum combustion in comparison with hydrocarbon fuels is the production of a bimodal size distribution of droplets (80-90\% of mass), consisting of sub-micron alumina smokes and alumina residues ranging roughly from 20 to $200 \mu \mathrm{m}$ depending on original aluminum particle size and degree of agglomeration.

The ignition of aluminum particles begins with a relatively short heterogeneous combustion stage and quickly transitions to a quasi-steady diffusion flame with a detached spherical flame positioned off the particle surface at two to five radii $[9,10]$. In addition to gaseous products from the flame, solid oxide condensates accumulate on the leeward side of the particle forming a cap. This cap reduces the overall surface area of the aluminum and results in violent surface gas ejections caused by both the dilution of molten aluminum with oxides and the participation of nitride reactions $[11,12]$.

The phenomena and mechanism of the aluminum particle combustion have been figured 
out through various analytical or experimental researches, and numerical models have been developed. Most of the previous studies centered on either ignition or quasi-steady combustion separately, and the rigorous numerical studies are computationally heavy to describe every details regarding the aluminum oxide.

The model [13] assumes that metal combustion is similar to droplet combustion, and is described using the $D^{2}$-law, and that ignition and combustion ought to depend on the melting and boiling points of the metal and the oxide. A model for the combustion of aluminum particles including oxidizer diffusion to the surface and heterogeneous reaction there is developed in [14]. Most reliable models are based on experimental and numerical correlations $[15,16]$. These models have focused on calculating the burning time and flame temperature of the aluminum particle based on the diffusion process, and inherently assume that the chemical kinetics is rapid enough to be ignored.

An unsteady two-dimensional evaporation-diffusion-kinetics controlled numerical model of the oxidation of aluminium particles is developed in [17]. The model takes into account the effects of forced convection, radiation, growth of an oxide lobe, variable transport properties and an extended condensation zone. A model for the burning of individual aluminum particles in gas mixtures containing several oxidizing reagents, which includes the buildup of oxide on an aluminum particle, evaporation kinetics of aluminum and surface chemical reactions is proposed in [18]. The model provides a good description of the experimental dependence of the burning time of aluminum particles on their size and major flow parameters.

A five-zone model that includes a more complete description of oxide cap formation, growth and movement is developed in [19]. This model is capable of capturing many of the observed dynamics of agglomerate motion. The effect of phase transformations in the aluminium oxide shell, which surrounds the metal particle, on the oxidation process is studied in [20]. Oxidation is considered to be a transport process through the oxide shell, and a multistage mechanism for oxidation of micron-sized aluminium powder is proposed. The model proposed in $[3,8]$ simulates the combustion of a single aluminum particle after ignition. The transition from the spherically symmetrical combustion mode to a non-symmetrical combustion mode with brightness oscillations, burning droplet speed variations, spinning smoke cloud shape, mini-jets and production of small secondary particle-satellites are observed experimentally in [21].

A simple model for the ignition and combustion of a single aluminum particle burning in air is presented in [22]. The model consists of two stages, ignition and combustion. In the ignition stage, melting and heterogeneous surface reactions are assumed to occur until a predefined transition temperature of the oxide is attained. In the combustion stage, a quasi-steady state diffusion flame is assumed accounting for the deposition of metal oxide on the surface of molten aluminum. Predictions of overall burning rate, particle velocity and flame radius show good agreement with experimental data. A quasi-steady model to predict instantaneous consumption of aluminum and generation of condensed oxide as a function of instantaneous particle size, ambient conditions, and cumulative amount of oxide in the cap is developed in [23].

Some empirical correlations, used in practice, take into account the burning rate, but only makes use of experimental results without physical interpretation [24]. The burning characteristics of fuel droplets containing nano- and micron-sized aluminum particles are investigated in [25]. Combustion of a single aluminum droplet in different atmospheres is simulated using a simplified approach with detailed models for the gas-phase and surface chemistry as well as molecular transport in [26]. A simplified analytical modelling of single aluminum particle combustion is conducted in [27]. Both the heat transfer from the hot ambient gas and the enthalpy of heterogeneous surface reaction served to cause the particle 
ignition. The role of heterogeneous reactions in aluminum combustion is discussed in [28].

A two-dimensional combustion model with detailed gas-phase kinetics is developed in [29] to investigate propellant flame structure. However, this model is computationally expensive and does not capture the complexity of an entire propellant matrix. A model which accounts not only for different particle sizes but also for their random distribution throughout the propellant is developed in [30]. Three-dimensional simulations of aluminized propellant combustion are presented in [31]. The simulations allow to examine the dynamics of aluminum particles in the near-neighborhood of the surface after detachment, and to provide an estimate of the time to ignition of the particles, and their speed and height above the surface at ignition. Two-phase flows with inert and burning aluminum particles as well as the influence of particles on acoustic oscillations in the combustion chamber are considered in $[32-35]$.

The assumption of the equilibrium character of phase transition is valid for large droplets leading to essential errors for small droplets. A mathematical model for non-equilibrium combustion of droplets in rocket engines is developed in [36]. This model allows to determine the divergence of combustion rate for the equilibrium and non-equilibrium models. Comparison of theoretical and experimental data shows that the non-equilibrium combustion of droplet plays an important role for small droplets leading to higher burning time.

Accurate modelling and simulation of the combustion and resulting regression of solid propellants entails research activities in several areas, including the description and propagation of the propellant combustion interface, modelling of solid propellant flames, combustion instability analysis, and constitutive modelling of energetic materials. A better understanding of the mechanism of oxidation of aluminium particle helps in predicting the combustion behavior of the energetic formulations in which these particles are added as an additive for enhanced rate of energy release. The effect of convection on the burning rate and ignition delay is unknown, but remains of significant concern. While many studies have centered on measuring and modelling burning rate characteristics of propellants, less efforts have been focused on aerodynamics, heat transfer and combustion of single aluminum droplet. One important phenomenon is the formation of an aluminium oxide cap moving on the particle surface during combustion and movement of particle. It causes particle asymmetry and obstructs part of the aluminium surface, leading to spinning [37]. Many of the models have not accounted for the effects of the oxide cap in the distortion of the symmetrical flame, and have concentrated on aluminum combustion in air.

The study focuses on the numerical analysis of flowfield around aluminium droplet with oxide cap inside combustion chamber of SRM. Geometry of the aluminium droplet and its oxide cap changes as mass is removed from the aluminium droplet, and oxide cap mass increases during aluminium combustion. The mathematical model of two-phase flow around a single aluminium droplet with oxide cap is developed and validated against experimental data. The model solves the continuity, momentum, energy and species continuity equations to obtain the species and temperature profiles and the burning time.

\section{Droplet combustion}

Aluminum particle burns in a gas-phase reaction where the flame front is at a detached distance away from the particle surface (Figure 1). The molten alumina coheres on the liquid aluminum surface and forms an oxide cap in a bulged shape owing to its higher surface tension. The oxide cap grows during quasi-steady combustion period as the reaction product diffuses inward and condenses, and the particle burning ends with the formation of 
a residue particle. A fraction of the oxide diffuses back and deposits on the particle surface and is termed as the oxide cap (lobe). The oxide cap tends to accumulate on the lower end of the particle. The accumulation of the oxide on the particle surface and the porosity of the oxide cap results in a final oxide cap size of the order of the initial particle size. The other fraction of the oxide is transported outwards and is termed as the oxide smoke. The oxide cap effect on the burning time depends on the initial size of the particle. The oxide cap acts as a dead weight which reduces the vaporization surface, leading to non-symmetrical combustion and a possible droplet spin.

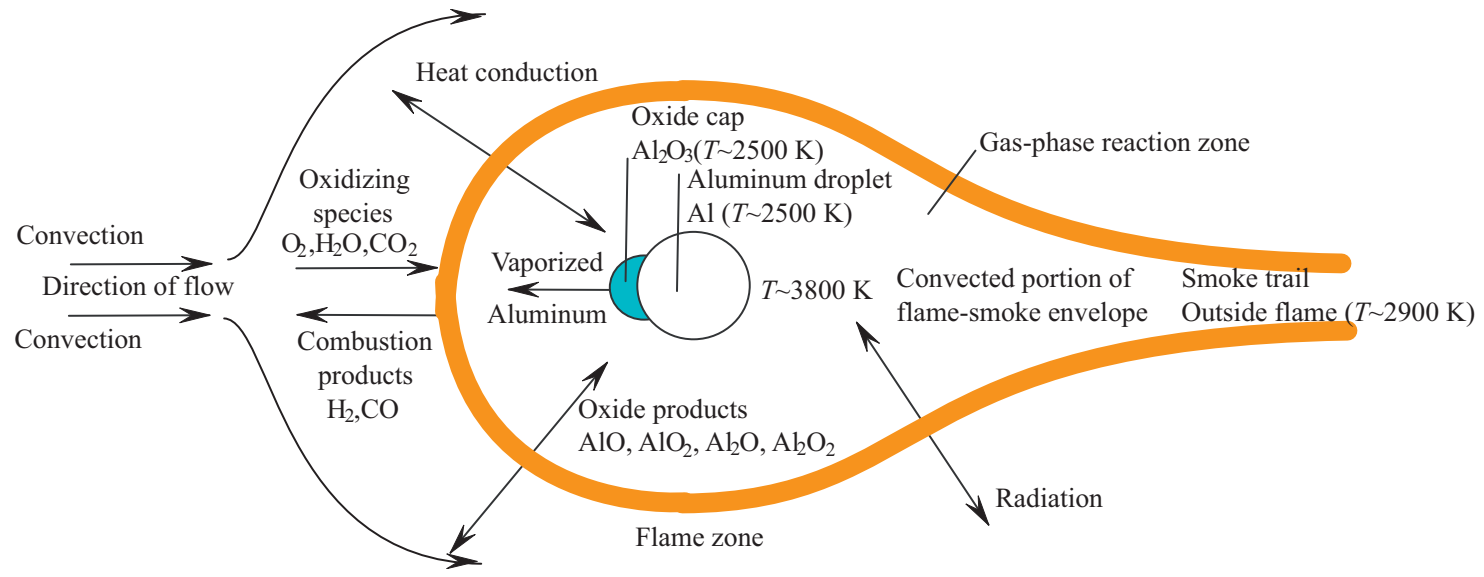

Figure 1. Aluminum particle combustion

Between surface temperature $(1200 \mathrm{~K})$ and initial temperature $(300 \mathrm{~K})$, two characteristic temperatures have to be considered: the aluminum melting temperature $(930 \mathrm{~K})$ and the binder temperature degradation. For particles with diameters greater than $100 \mu \mathrm{m}$, the majority of experimental studies have shown that ignition takes place at a temperature near the melting point of aluminum oxide $(2300 \mathrm{~K})$. Since each aluminum particle is covered by an impervious oxide shell, it was argued that the particle does not ignite until the oxide shell melts or breaks up near its melting temperature under the effect of aluminum thermal expansion. As an aluminum particle is heated to the melting temperature, the aluminum melts but is still contained within the oxide shell. Further heating causes the liquid aluminum to develop vapor pressure within the shell. When the particle temperature approaches the melting point of the oxide, oxide shell weakens and eventually breaks. At this point, the aluminum vapor is released from within the shell and diffuses into the surrounding atmosphere to begin reacting with the available oxidizing species. This condition defines the ignition point of the aluminum and the beginning of the combustion process.

Because the oxide melting temperature is higher than the melting temperature of aluminum, the aluminum is assumed to be fully melted upon ignition. The molten aluminum particle surface burns at a temperature between the oxide melting temperature, and the aluminum boiling temperature $[10,16]$. Although the global oxidation reaction of aluminum produces alumina, $\mathrm{Al}_{2} \mathrm{O}_{3}$, many sub-oxides such as $\mathrm{AlO}, \mathrm{AlO}_{2}, \mathrm{Al}_{2} \mathrm{O}$ and $\mathrm{Al}_{2} \mathrm{O}_{2}$ exist in the flame-zone, with $\mathrm{AlO}$ as the most prominent. The condensation of alumina at its estimated boiling point $(3800 \mathrm{~K})$ sets the approximate temperature of the oxide cloud because $\mathrm{Al}_{2} \mathrm{O}_{3}(\mathrm{l})$ condenses directly from the association of the sub-oxides and the idealized gaseous form, $\mathrm{Al}_{2} \mathrm{O}_{3}(\mathrm{~g})$, immediately dissociates back into $\mathrm{AlO}$ and $\mathrm{O}_{2}$ species $[17,38]$. 


\section{Key processes}

Particulate phase contains sub-micron alumina smoke that is modelled as a particular gas component, and agglomerates with alumina residues that are carried out into the flowfield. Size of agglomerates depends on the size of original aluminium particles and the degree of agglomeration in the near-surface layer of composite propellant. It has been assumed that the oxide deposits uniformly on the particle surface and migrates to the downstream side to coalesce into an oxide cap.

Motion of aluminum droplet with oxide cap in fluid flow is defined by relative Reynolds number and relative Weber number

$$
\operatorname{Re}_{p}=\frac{2 R_{p} \rho\left|\boldsymbol{v}_{g}-\boldsymbol{v}_{p}\right|}{\mu}, \quad \mathrm{We}_{p}=\frac{2 R_{p} \rho\left|\boldsymbol{v}_{g}-\boldsymbol{v}_{p}\right|^{2}}{\sigma},
$$

where $R_{p}$ is the effective radius of particle, $\rho$ is the fluid density, $\mu$ is the fluid dynamic viscosity, and $\sigma$ is the droplet surface tension. For SRM conditions, $\rho=5.88 \mathrm{~kg} / \mathrm{m}^{3}, p=1-$ $20 \mathrm{MPa},\left|\boldsymbol{v}_{g}-\boldsymbol{v}_{p}\right| \sim 5 \mathrm{~m} / \mathrm{s}, \mu=6.7 \times 10^{-5} \mathrm{~Pa} \times \mathrm{s}, \sigma=0.84 \mathrm{~N} / \mathrm{m}$. The relative Reynolds number based on droplet diameter varies from 100 near the propellant surface, where the burning gases have the greatest velocity, to zero when can be assumed that a Stokes regime is reached. This interval includes different flow regimes around the sphere, but flow around an aluminum droplet is laminar due to the small size of the droplet (diameter of droplet is less than $200 \mu \mathrm{m})$.

Compared to motion of solid particles, motion of liquid droplets is accompanied by the deformation of their shape at low Weber numbers and their break-up under the action on aerodynamic forces at high Weber numbers. At low relative Reynolds numbers $\left(\operatorname{Re}_{p}<100\right)$, modification of droplet drag due to its deformation is caused by increasing its cross sectional area. A ratio of midlength section radius of deformed droplet, $R_{M}$, to initial radius of droplet, $R_{0}$, is a function of relative Weber number, $R_{M} / R_{0}=1+0.03 \mathrm{We}_{p}$. For low Weber numbers $\left(\mathrm{We}_{p}<1\right)$, increase in cross sectional area of droplet due to its deformation is less than $3 \%$. Deformation of droplet ant its break-up is taken into account in long channels $(L / R>30)$, in which Weber number reaches critical value $\left(\mathrm{We}_{*}=18-23\right)$.

Aerodynamic shear induces internal circulation in droplets (Figure 2). This liquid motion continually sweeps fresh fluid to the surface of the droplet where it is available for oxidation. The oxide that forms at the droplet surface is entrained into the particle and mixed with the liquid aluminum by convective motion within the droplet. The internal circulation of the in-flight molten aluminum droplets affects the amount of oxides in the droplets. A toroidal flow induced inside droplet leads to increase in the final oxidation percentage of a typical coating increases as compared to a droplet without internal circulation [39].

The oxide volume fraction within a droplet with internal circulation is about $8 \%$ [39]. The oxide content calculated for an average droplet with internal circulation correlates well with the experimentally determined oxide content from 3.3 to $12.7 \%$. In contrast, the oxide volume fraction computed for a rigid sphere is nearly two orders of magnitude smaller than that for a droplet with internal circulation. The oxide buildup on the surface of a rigid sphere under the same flight conditions is estimated to be $0.121 \%$, which is approximately $1.5 \%$ of the oxide volume fraction for a droplet with internal circulation. The analysis, performed in [39], shows that internal circulation within the droplet increases the oxide content of the droplet by two orders of magnitude over that of a droplet without internal circulation. Time of particle heating to boiling temperature is less than burning time of droplet, therefore the droplet temperature is uniform, and it equals to boiling temperature.

The correction to drag of liquid droplet due to internal recirculation motion has an order 


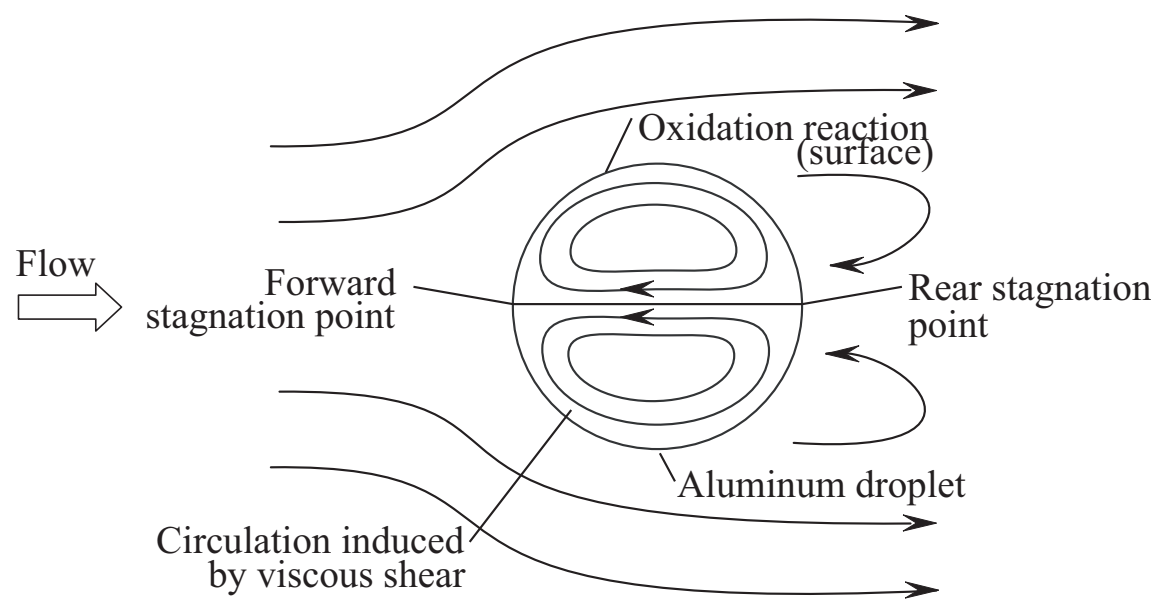

Figure 2. Internal circulation within a fully molten droplet caused by shear forces from the high-velocity airflow around the droplet

of ratio of dynamic viscosity of fluid, $\mu$, to dynamic viscosity of liquid, $\mu_{A l}$. For aluminum droplet $\mu / \mu_{A l} \ll 1$, therefore velocity of internal recirculation motion is less than velocity of external fluid flow, and does not affect droplet drag and heat transfer.

\section{Geometry of droplet}

The droplet is represented by a constant-diameter sphere with a segment occupied by the oxide cap to reduce the reactive surface. The shape of droplet depends on mass fraction of aluminium and temperature. The mass is removed from the aluminium droplet and the mass of oxide cap increases during combustion. It is assumed the aluminum droplet is spherical with radius $R_{1}$, and the oxide cap represents a segment of sphere with radius $R_{2}$ which intersects the droplet surface at a contact angle $\theta$ (Figure 3). The droplet temperature is uniform and equal to the boiling point. The oxide deposits uniformly on the droplet and migrates to the downstream side to coalesce into oxide cap.

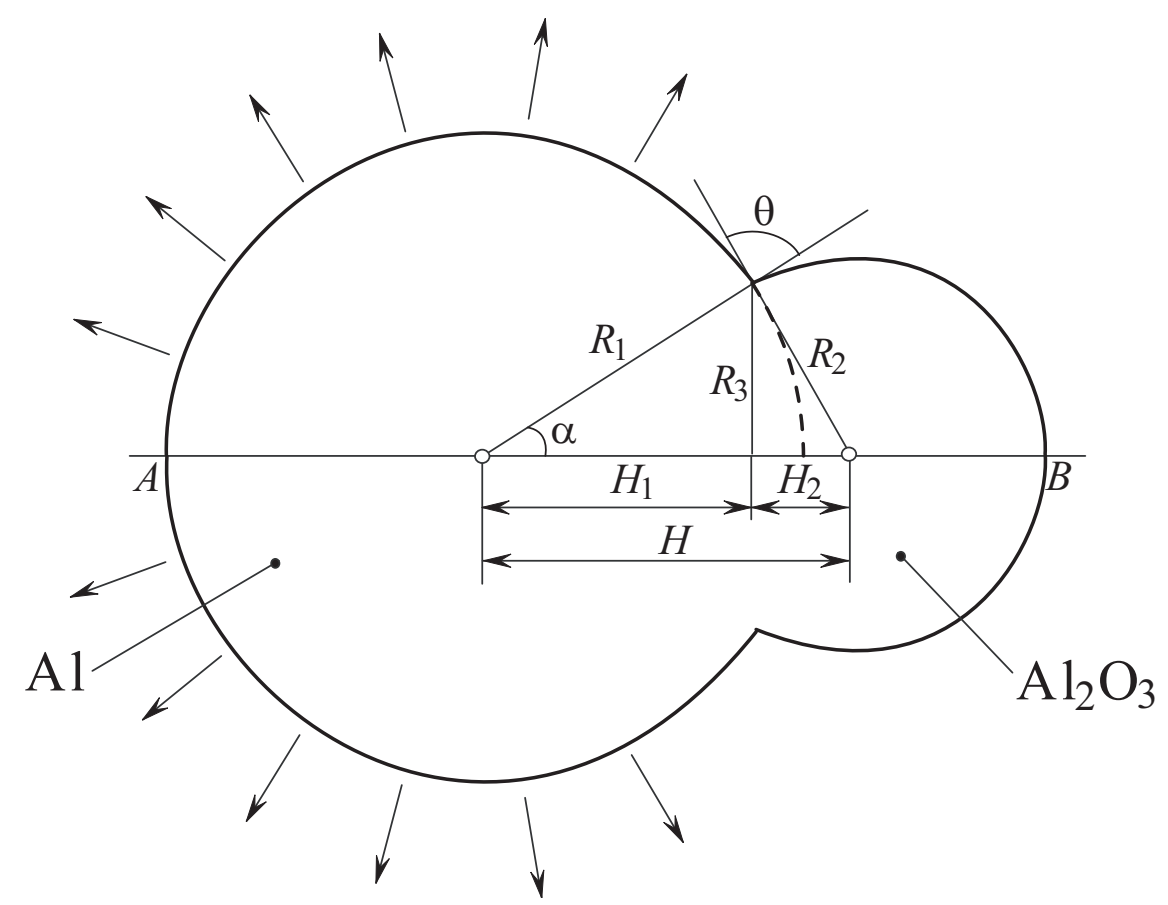

Figure 3. Geometry of aluminum droplet with oxide cap 
The oxide cap inhibits aluminum vaporization from the portion of the sphere it covers. This is because the oxide has almost twice the density of the metal and so the metal cannot diffuse through the oxide. The oxide cap growth and the formation of the residue particle are expressed in terms of the mass of aluminum oxide deposited on the particle surface only. Geometry of the aluminum droplet and its oxide cap changes as mass is removed from the aluminum, and oxide cap mass increases during aluminum combustion. For given instantaneous conditions, droplet size, oxide cap size and fractional aluminum surface area coated by the oxide cap are calculated with the use an iterative approach to provide correct values. The contact angle is found from the Young's equation

$$
\cos \theta=\frac{\sigma_{s v}-\sigma_{s l}}{\sigma_{l v}}
$$

where $\sigma_{s v}, \sigma_{l v}$ and $\sigma_{s l}$ are the surface tensions of solid-vapor $\left(\mathrm{Al}_{2} \mathrm{O}_{3}\right.$ and vapor), liquidvapor ( $\mathrm{Al}$ and vapor) and solid-liquid $\left(\mathrm{Al}_{2} \mathrm{O}_{3}\right.$ and $\left.\mathrm{Al}\right)$ surfaces. The surface tensions are the functions of temperature. This way is more sophisticated than the simplified geometrical model of aluminum droplet used in [23], in which the angle of intersection of two spheres is fixed at 90 degrees.

With a given contact angle, the shape of agglomerate's cap is described by the radius of aluminum droplet, $R_{1}$, the radius of oxide cap, $R_{2}$, the radius of circle of intersection of two spheres, $R_{3}$, the distance between the centers of aluminum droplet and oxide cap, $H$, and two parameters, $H_{1}$ and $H_{2}$, which are the heights of sphere segments. The intersection of the spheres is a circle with radius

$$
R_{3}=\frac{R_{1} R_{2}}{H} \sin \theta
$$

The distance between the centers of the spheres is related to the aluminum droplet radius and the oxide lobe radius

$$
H=R_{1}^{2}+R_{2}^{2}-2 R_{1} R_{2} \cos \theta
$$

For the triangle formed with the sides $R_{1}, R_{2}$ and $H$, the following relationships take place

$$
R_{1}^{2}-H_{1}^{2}=R_{3}^{2}, \quad R_{2}^{2}-H_{2}^{2}=R_{3}^{2}, \quad H_{1}+H_{2}=H .
$$

The linear angle is then calculated as

$$
\alpha=\arcsin \left(\frac{R_{3}}{R_{1}}\right) .
$$

The solid angle is

$$
\vartheta=2 \pi(1-\cos \alpha) .
$$

The fraction of the aluminum sphere radius covered with oxide (fractional blockage of surface) is calculated as

$$
\beta=\frac{\vartheta}{4 \pi}=\frac{1}{2}(1-\cos \alpha) .
$$

The mass of aluminum droplet with oxide cap is a sum of mass of aluminum droplet and mass of oxide cap

$$
m=m_{A l}+m_{A_{2} O_{3}} .
$$


The effective total particle radius (liquid metal plus metal oxide) is defined as

$$
R_{M}=\left[\frac{3}{4 \pi}\left(\frac{m_{A l}}{\rho_{A l}}+\frac{m_{A l_{2} O_{3}}}{\rho_{A l_{2} O_{3}}}\right)\right]^{1 / 3} .
$$

The mass fraction of aluminum and the mass fraction of alumina are

$$
C_{A l}=\frac{m_{A l}}{m_{A l}+m_{A l_{2} O_{3}}}, \quad C_{A l_{2} O_{3}}=\frac{m_{A l_{2} O_{3}}}{m_{A l}+m_{A l_{2} O_{3}}},
$$

where $C_{A l}+C_{A_{2} O_{3}}=1$. The average density of aluminum droplet with oxide cap is

$$
\rho=\left(\frac{C_{A l}}{\rho_{A l}}+\frac{1-C_{A l}}{\rho_{A l_{2} O_{3}}}\right)^{-1} .
$$

The mass of aluminum droplet and the mass of oxide cap are the products of volume and density

$$
m_{A l}=\rho_{A l} V_{A l}, \quad m_{A_{2} O_{3}}=\rho_{A l_{2} O_{3}} V_{A l_{2} O_{3}} .
$$

The volume of aluminum droplet is found as the volume of sphere with radius $R_{1}$ by

$$
V_{A l}=\frac{4}{3} \pi R_{1}^{3}
$$

The radius of aluminum droplet is

$$
R_{1}=\left(\frac{3 m_{A l}}{4 \pi \rho_{A l}}\right)^{1 / 3} .
$$

The volume of alumina cap is found as a difference between the volume of sphere segment with radius $R_{3}$ and height $R_{2}+H_{2}$, and volume of sphere segments with radius $R_{3}$ and height $R_{1}-H_{1}$ by

$$
V_{A l_{2} O_{3}}=\frac{\pi}{6}\left(R_{2}+H_{2}\right)\left[3 R_{3}^{2}+\left(R_{2}+H_{2}\right)^{2}\right]-\frac{\pi}{6}\left(R_{1}-H_{1}\right)\left[3 R_{3}^{2}+\left(R_{1}+H_{1}\right)^{2}\right] .
$$

The mass of aluminum droplet and the mass of oxide cap are related with the equation

$$
\begin{aligned}
& 8 \frac{1-C_{A l}}{C_{A l}} \frac{\rho_{A l}}{\rho_{A l_{2} O_{3}}} R_{1}^{3}= \\
& =\left(R_{2}+H_{2}\right)\left[3 R_{3}^{2}+\left(R_{2}+H_{2}\right)^{2}\right]-\frac{\pi}{6}\left(R_{1}-H_{1}\right)\left[3 R_{3}^{2}+\left(R_{1}+H_{1}\right)^{2}\right] .
\end{aligned}
$$

Since the cap radius is not initially known, but volume and mass of the oxide cap are known via the cumulative oxide cap mass accumulation, an iterative procedure of solution of equations from (1) to (4) is used to find a cap radius for a given values of the aluminum sphere radius and aluminum fraction which gives the correct value for volume of the oxide cap. However, only aluminium evaporation is modelled by assuming the equilibrium vapor fraction at the droplet surface.

The particle usually ignites near the propellant surface. Hence, ignition is assumed to have occurred initially, and the model concentrates on the combustion after the ignition. Since ignition is taken to have occurred, the initial particle surface temperature is taken to be the boiling point of aluminum at the prevailing pressure. Assuming a liquid-vapor equilibrium, the partial pressure of the aluminum sub-oxides and aluminum oxides is expressed 
using the Clausius-Clayperon equation. Thermodynamic properties of any species are defined by specifying a molar mass, a temperature polynomial for the molar heat capacity at constant pressure as well as molar enthalpy of formation and entropy of formation.

The rate of heterogeneous surface reaction is expressed in an Arrhenius-type of formulation with experimentally determined pre-exponent coefficient and activation energy [40]. Occurrence of the reaction is valid only after the initiation of the melting.

The droplet surface is modelled as either reactive or non-reactive. For the reactive droplet surface, the boundary conditions cannot be directly specified in terms of physical quantities. The surface conditions are determined by satisfying balance relations for mass and thermal fluxes. Among the bulk species, liquid aluminum is the only one which is consumed by the surface reactions and its fraction in the droplet is taken equal to 1 by neglecting the other bulk species generated by the surface reactions. For the non-reactive droplet surface, only aluminum evaporation is taken into account by assuming equilibrium between gaseous and liquid aluminum on the surface.

Burning time is one of the primary characteristics of droplet combustion, which is often represented as a function of the initial droplet diameter $D_{0}$ as $t_{b}=a D_{0}^{n}$. The value of $n$ depends on the combustion regime: $n=2$ for diffusion-controlled combustion with a detached gaseous flame around the droplet and $n=1$ for combustion controlled by heterogeneous kinetics on the droplet surface. For aluminum particle combustion, an empirical correlation with $n=1.8$ and a coefficient dependent on the pressure, temperature, and fractions of oxidizer species is proposed in [3].

\section{$5 \quad$ Governing equations}

The model solves the continuity, momentum, energy and species continuity equations simultaneously to obtain the species and temperature profiles and the burning time. In Cartesian coordinates $(x, y, z)$, an unsteady three-dimensional flow of a gas mixture is described by the following equation

$$
\frac{\partial Q}{\partial t}+\frac{\partial F_{x}}{\partial x}+\frac{\partial F_{y}}{\partial y}+\frac{\partial F_{z}}{\partial z}=S
$$

Equation (5) is complemented with the equation

$$
p=(\gamma-1) \rho\left[e-\frac{1}{2}\left(v_{x}^{2}+v_{y}^{2}+v_{z}^{2}\right)\right] .
$$

The vector of conservative variables, $Q$, and the flux vectors, $F_{x}, F_{y}$ and $F_{z}$, have the following form

$$
Q=\left(\begin{array}{c}
\rho \\
\rho v_{x} \\
\rho v_{y} \\
\rho v_{z} \\
\rho e \\
\rho Y_{O} \\
\rho Y_{F}
\end{array}\right)
$$




$$
\begin{aligned}
& F_{x}=\left(\begin{array}{c}
\rho v_{x} \\
\rho v_{x} v_{x}+p-\tau_{x x} \\
\rho v_{x} v_{y}-\tau_{x y} \\
\rho v_{x} v_{z}-\tau_{x z} \\
(\rho e+p) v_{x}-v_{x} \tau_{x x}-v_{y} \tau_{x y}-v_{z} \tau_{x z}+q_{x} \\
\rho Y_{O} v_{x}+G_{O x} \\
\rho Y_{F} v_{x}+G_{F x}
\end{array}\right) \\
& F_{y}=\left(\begin{array}{c}
\rho v_{y} \\
\rho v_{y} v_{x}-\tau_{y x} \\
\rho v_{y} v_{y}+p-\tau_{y y} \\
\rho v_{y} v_{z}-\tau_{y z} \\
(\rho e+p) v_{y}-v_{x} \tau_{y x}-v_{y} \tau_{y y}-v_{z} \tau_{y z}+q_{y} \\
\rho Y_{O} v_{y}+G_{O y} \\
\rho Y_{F} v_{x}+G_{F y}
\end{array}\right) \\
& F_{z}=\left(\begin{array}{c}
\rho v_{z} \\
\rho v_{z} v_{x}-\tau_{z x} \\
\rho v_{z} v_{y}-\tau_{z y} \\
\rho v_{z} v_{z}+p-\tau_{z z} \\
(\rho e+p) v_{z}-v_{x} \tau_{z x}-v_{y} \tau_{z y}-v_{z} \tau_{z z}+q_{z} \\
\rho Y_{O} v_{z}+G_{O z} \\
\rho Y_{F} v_{z}+G_{F z}
\end{array}\right) .
\end{aligned}
$$

The source term is

$$
S=\left(J, 0,0,0, H, R_{O}, R_{F}\right)^{\prime}
$$

Here, $t$ is the time, $\rho$ is the density, $v_{x}, v_{y}$, and $v_{z}$ are the velocity components in the coordinate directions $x, y$, and $z, p$ is the pressure, $e$ is the total energy per unit mass, $T$ is the temperature, $Y_{O}$ is the mass fraction of oxidizer $\left(Y_{O}=\rho_{O} / \rho\right), Y_{F}$ is the mass fraction of fuel $\left(Y_{F}=\rho_{F} / \rho\right)$, and $\gamma$ is the specific heat capacities ratio.

The viscous stress tensor and heat flux are found from the Newton's law and Fourier law

$$
\tau_{i j}=\mu\left(\frac{\partial v_{i}}{\partial x_{j}}+\frac{\partial v_{j}}{\partial x_{i}}-\frac{2}{3} \frac{\partial v_{k}}{\partial x_{k}} \delta_{i j}\right), \quad q_{i}=-\lambda \frac{\partial T}{\partial x_{i}} .
$$

The diffusive fluxes of oxidizer and fuel are defined by Fick's law

$$
G_{O i}=-D \frac{\partial Y_{O}}{\partial x_{i}}, \quad G_{F i}=-D \frac{\partial Y_{F}}{\partial x_{i}}
$$

The thermal conductivity and the diffusion coefficient are expressed as $\lambda=c_{p} \mu / \operatorname{Pr}$ and $D=c_{p} \mu / \mathrm{Sc}$, where $c_{p}$ is the specific heat capacity at constant pressure, $\mathrm{Pr}$ is the Prandtl number, and Sc is the Schmidt number.

The source therm in the continuity equation is

$$
J_{k}=M_{k} \omega_{k},
$$


where $M_{k}$ is the molar mass, and $\omega_{k}$ is the molar production rate of species. The production rate for the $k$ th species is defined as

$$
\omega_{k}=\sum_{i=1}^{I} \chi_{k i} u_{i}
$$

The stoichiometric coefficient is

$$
\chi_{k i}=n_{k i}^{\prime \prime}-n_{k i}^{\prime}
$$

Rate of progress variable is

$$
u_{i}=k_{f i} \prod_{k=1}^{K} \chi_{k i}^{n_{k i}^{\prime}}-k_{r i} \prod_{k=1}^{K} \chi_{k i}^{n_{k i}^{\prime \prime}} .
$$

For each reaction $i$, the forward and reverse constants, $k_{f i}$ and $k_{r i}$, are both described by an Arrhenius law

$$
k=A T^{\beta} \exp \left(-\frac{E}{R T}\right)
$$

Oxidizer rate and evaporation products rate are defined as follows

- If $Y_{O}<\chi Y_{F}$ then

$$
R_{O}=-4 \pi Y_{O} \mu \sum_{l=1}^{L} n_{p}^{l} r_{p}^{l}, \quad R_{F}=\frac{R_{O}}{\chi} .
$$

- If $Y_{O} \geq \chi Y_{F}$ then

$$
R_{F}=-4 \pi Y_{F} \mu \sum_{l=1}^{L} n_{p}^{l} r_{p}^{l}, \quad R_{O}=\frac{R_{F}}{\chi} .
$$

In the above expressions, $\chi$ is the stoichiometric ratio of oxidizer and fuel. Gas phase rate is a sum of oxidizer rate and evaporation products rate

$$
J=R_{O}+R_{F} .
$$

Heat release per unit volume of mixture as a consequence of condensation is a product of fuel rate, $R_{F}$, and heat of condensation, $Q_{c}$, therefore

$$
H=R_{F} Q_{c}
$$

The radius of oxide particle of fraction $l, r_{p}^{l}$, and the number of oxide particles per unit volume, $n_{p}^{l}$, are calculated from the solution of equations describing motion of oxide particles (these equations are written in Eulerian coordinates).

Small oxide particles follow streamlines of fluid flow, and their trajectories are calculated as a small perturbation of streamlines of fluid flow. Condensation rate of particle of fraction $l$ is

- If $Y_{O} \geq \chi Y_{F}$ then

$$
J^{l}=4 \pi \mu r_{p} Y_{F}(1+\chi) .
$$


- If $Y_{O}<\chi Y_{F}$ then

$$
J^{l}=4 \pi \mu r_{p} Y_{F}\left(\frac{1+\chi}{\chi}\right) .
$$

The transport properties are defined by specifying parameters of the potential function for pure species and by applying the classical formalism of the molecular kinetics theory.

At the initial time moment $(t=0)$, the droplet is heated uniformly. Uniform distribution of the particles of smoke oxide in the computational domain is assumed. The velocity, temperature, fractions of oxidizer and fuel are specified on the inlet boundary of computational domain. Velocity, temperature, oxidizer fraction and fuel fraction are specified on a free surface of aluminum droplet $\left(v_{\tau}=0, v_{n}=v_{w}, T=T_{s}, \partial Y_{O} / \partial n=0, Y_{F}=Y_{F 0}\right.$, where $v_{w}$ is the velocity of injection calculated from the thermal balance equation on a free surface of aluminum droplet). If droplet is heated non-uniformly, then the thermal balance equations has the following form

$$
m h_{L}+\left(\lambda \frac{d T}{d r}\right)_{s}=\left(\lambda \frac{d T}{d r}\right)_{g},
$$

where $m=\rho v_{w}$ is the mass flow rate, $h_{L}$ is the specific enthalpy of phase transition. There is no evaporation from the area of the droplet covered by the oxide cap. Velocity, temperature, oxidizer fraction and fuel fraction are specified on a surface of oxide cap where heterogeneous oxidation of aluminum takes place $\left(v_{\tau}=0, v_{n}=v_{O}, T=T_{s}, Y_{O}=0, \partial Y_{F} / \partial n=0\right.$, where $v_{O}$ is the velocity of oxidizer).

\section{$6 \quad$ Numerical method}

The calculations are based on in-house finite volume unstructured CFD code using an edgebased data structure to give the flexibility to run on meshes composed of a variety of cell types [41]. This has the advantage that hexahedral or prismatic elements can be used to capture velocity gradients through the boundary layer adjacent to a surface. At the same time, hybrid meshes can incorporate pyramid and tetrahedral cells in the freestream regions to provide greater flexibility to represent complex geometries.

The solver works in an explicit time-marching fashion, based on a three-step RungeKutta stepping procedure. Convergence to a steady state is accelerated by the use of multigrid techniques, and by the application of block-Jacobi preconditioning for high speed flows, with a separate low Mach number preconditioning method for use with low speed flows. The sequence of meshes is created using an edge-collapsing algorithm. Preconditioning improves the rate at which information propagates through the flow domain during the solution iterations.

Although experimental results have indicated that the flame zone is within a distance of 10 particle radii, the calculation domain for this model covers 60 particle radii to ensure that the input conditions are totally unaffected by the combustion. A fine mesh is required to reproduce a sharp phase interface. A mesh sensitivity analysis is performed using a mesh with 4 million (fine mesh, mesh 1), 2 million (intermediate mesh, mesh 2) and 1 million (coarse mesh, mesh 3) of cells. The results with 2 million of cells are similar to those with 4 million of cells. The simulations are performed using a mesh consisting of 2 million of cells, and mesh 2 is used in CFD simulations of flowfield around inert and reactive droplet. Use of coarse mesh leads to significant errors in estimations of drag coefficient and burning time 

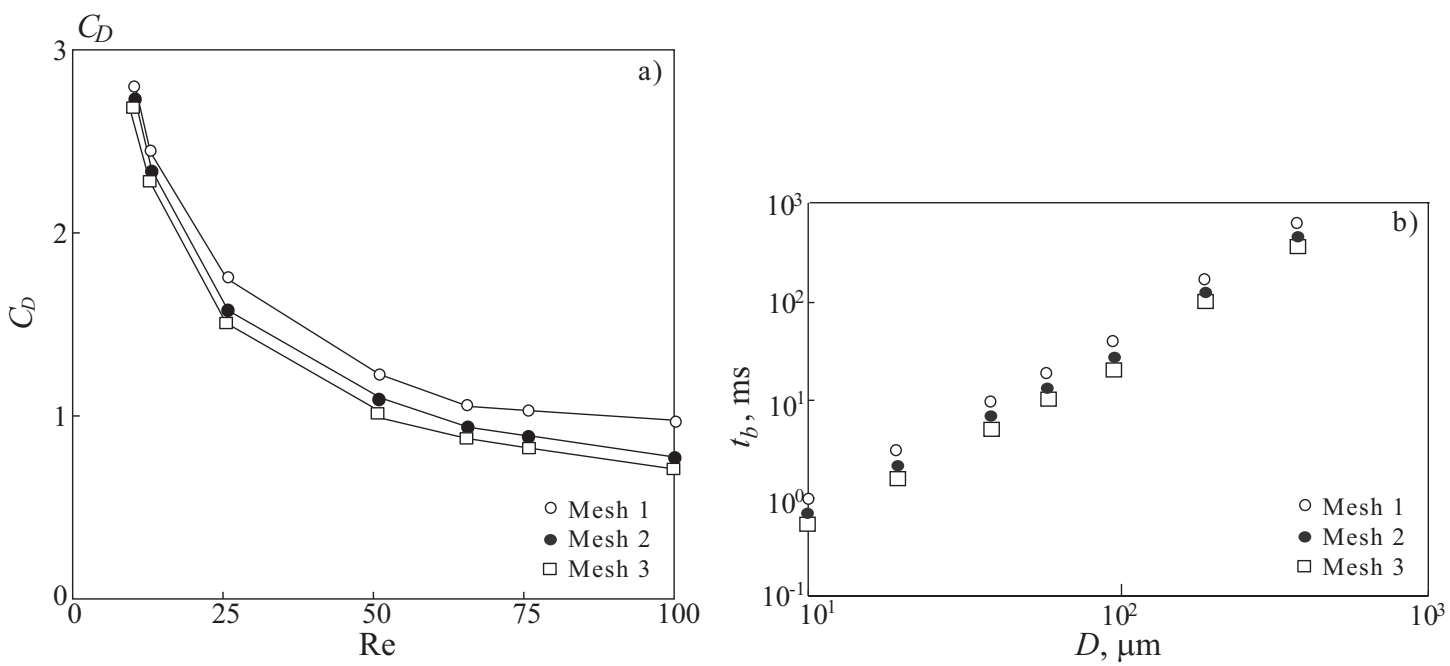

Figure 4. Dependence of drag coefficient (a) and burning time (b) on mesh resolution for coarse mesh (symbols $\circ$ ), intermediate mesh (symbols • ) and fine mesh (symbols $\square$ )

of aluminium droplet, and discrepancy of the results computed with coarse and fine mesh increases with increasing of the Reynolds number and droplet diameter (Figure 4).

Depending on spatial resolution and numerical solver used a definite error occurs in integration at each step. This error is expressed in absolute or relative values. Decreasing the length scale to infinitely small values brings to minimization of accumulated error for convergent schemes. However, the real simulation operates with final cell size thus introducing a definite limit to an allowable number of integration steps, until accumulated error exceeds acceptable value. In case of systematic error being the defect of CFD solver, accumulation of error is proportional to number of steps.

Solving numerical problems it is necessary to control accumulation of numerical error. For fine mesh simulations of unsteady processes numerical time step is chosen from the Courant condition, which makes it small, and the number of time steps is enormous. The numerical simulation precision and stochastic errors accumulation in solving problems of combustion and detonation of gas mixtures in rocket engines is studied in [42] based on the mathematical model developed and investigation of the influence of mesh size on computational precision and speed is performed. Accumulation of numerical errors in CFD simulation is controlled based on the algorithm described in [42].

Accumulation of errors takes place in a fast manner for coarse mesh and decreases on increasing mesh resolution. Reliability of results increases on increasing mesh resolution and scheme accuracy, but decreases on increasing physical time. The results computed demonstrate high reliability of the used CFD solver, but it is not always the case for simulating longer time periods. The computational errors accumulate faster for coarse mesh despite of the fact that overall simulation process needs much less time steps. Reliability of results increases on increasing mesh resolution and scheme accuracy, but decreases on increasing physical time of the process.

\section{Results and discussion}

Calculations are performed over a $100 \mu \mathrm{m}$ droplet in combustion. The surface temperature was assumed to be at the aluminum boiling temperature for pressure of 1 atm $(2750 \mathrm{~K})$. 


\subsection{Flow around droplet}

Axisymmetric flow is simulated around a constant-diameter aluminum droplet with and without oxide cap in order to verify and validate mesh resolution and the numerical scheme.

Streamlines around droplet with oxide cap are shown in the Figure 5. The vortex wake behind droplet is formed at Reynolds number of 10, and change of flow regimes takes place at lower Reynolds numbers compared to the fluid flow around rigid sphere. Flow around sphere is symmetrical relative to meridional plane and stationary at $\operatorname{Re}<210$. Transition to non-symmetrical and stationary flow is observed at Reynolds numbers between 210 and 250. If Reynolds number lies between 250 and 380, periodical oscillation of weak behind a sphere take place. Vortex road behind a sphere rises if $\operatorname{Re}>380$, and flow around sphere is non-stationary and non-symmetrical.

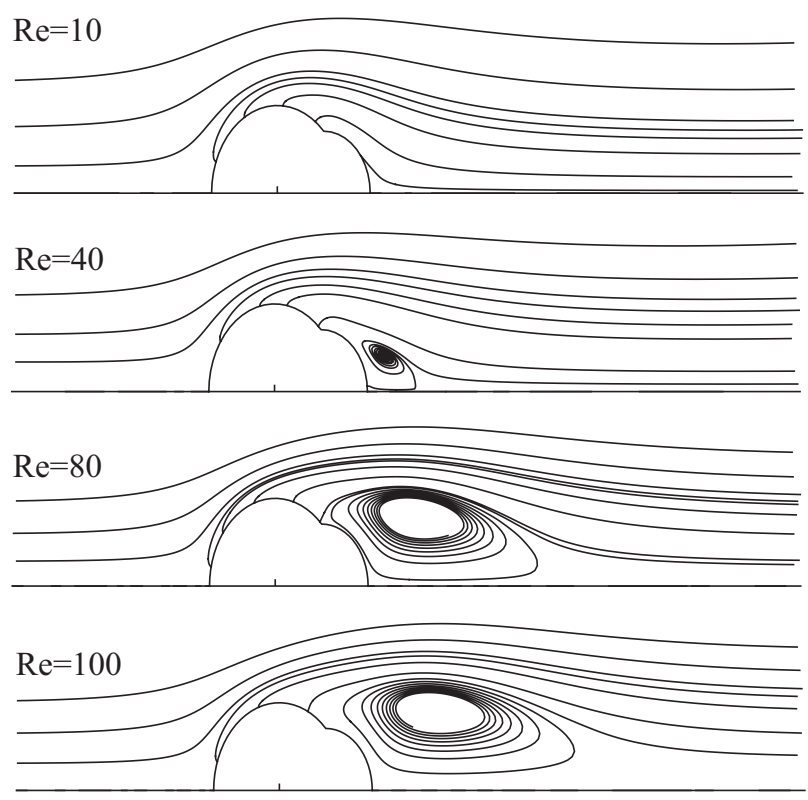

Figure 5. Streamlines around droplet with oxide cap at different Reynolds numbers

The determination of the sphere drag coefficient seemed to be a reasonable manner to verify the accuracy of the aerodynamic field near the droplet surface. For the purposes of comparison, experimental law is used (symbols o), as it is shown in the Figure 6. Calculated drag coefficient (symbols $\bullet$ ) for various Reynolds numbers is found to agree correctly with this correlation. The difference remains of the order of $5 \%$ which represents the average incertitude of the standard curve.

To investigate non-symmetrical flow around sphere to addition to flow at high Reynolds numbers, linear velocity profile on inlet boundary was specified $U=U_{a}+\beta x$, where $\beta$ is shear parameter $(\beta>0)$. Influence of Reynolds number and shear parameter on drag coefficient and lift coefficient is estimated. Dependencies of lift coefficient on shear parameter and Reynolds number are presented in the Figure 7. Flow around sphere is non-symmetrical at all Reynolds numbers. Oscillations of vortex wake start at $\beta=0.2$ and $\operatorname{Re}=230$. Increase in shear parameter leads to decrease in Reynolds number corresponding to transition from one flow regime to other. It is interesting to note the sign reversal of lift force at specific Reynolds number $\mathrm{Re}_{*}$ (in particularly, this specific Reynolds number is about 50 at $\beta=0.2$ ). The lift force is directed to the side where flow velocity is less if $\operatorname{Re}<\operatorname{Re}_{*}$, and lift force acts in opposite direction if $\mathrm{Re}>\mathrm{Re}_{*}$. Contribution of pressure forces is positive if $\operatorname{Re}<100$, and it is larger than contribution of viscous forces, so $C_{L}>0$. Increase in Reynolds number 


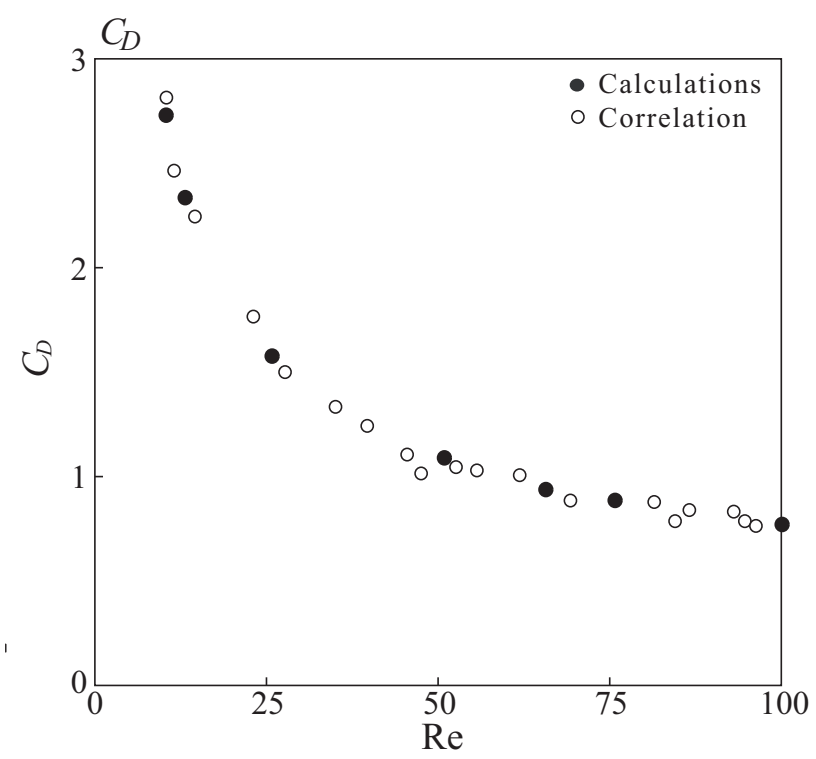

Figure 6. Drag coefficient as function of Reynolds number. Comparison of calculations (solid line) with the standard law (symbols $\bullet$ )

leads to negative contribution of pressure forces, and it prevails contribution of viscous forces if $\operatorname{Re}>200$. For $\operatorname{Re}>300$, the lift coefficient is approximately independent on shear parameter. Contribution of viscous forces decreases linearly at all Reynolds numbers as shear parameter increases. Drag coefficient increases if shear parameter increases at fixed Reynolds number.
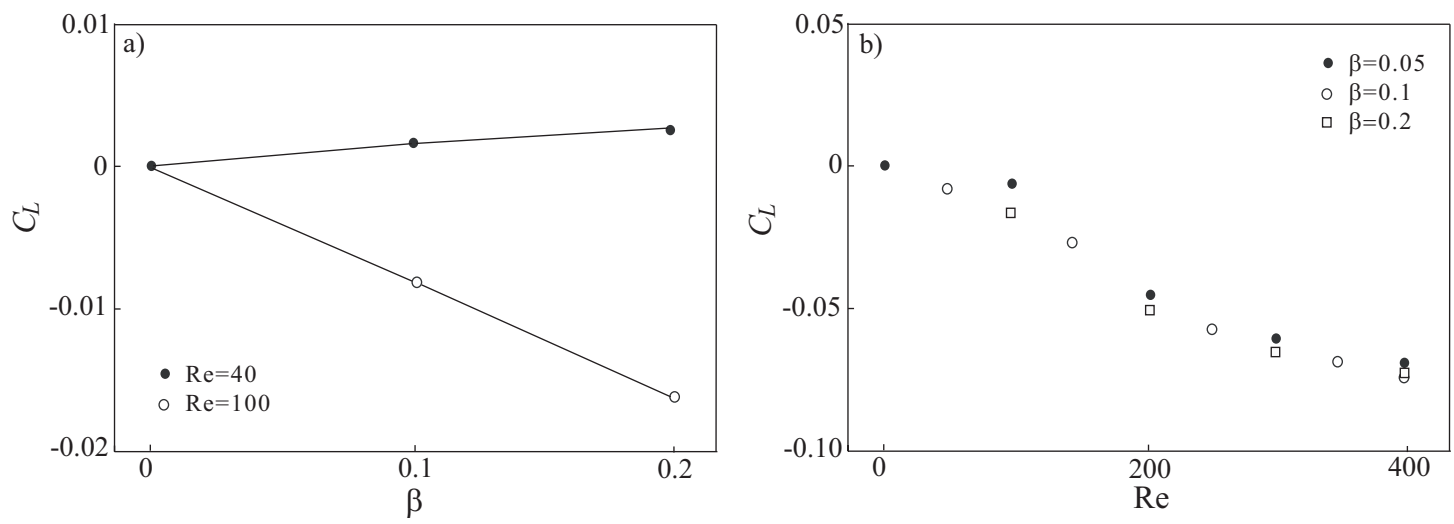

Figure 7. Dependence of lift coefficient on shear parameter and Reynolds number

Dependence of lift coefficient on time is presented in the Figure 8. Flow around sphere is stationary and symmetrical if $\beta=0$. Increase in shear parameter leads to weak oscillations of vortex wake behind the sphere (vortex wake is not observed), but these oscillations have a small influence on sphere drag. Vortex wake behind the sphere takes place at $\beta \sim 0.1$. Increase in shear parameter up to $\beta \sim 0.2$ causes increase in velocity amplitude in the weak, but flow remains regular. Regime flow depends on Reynolds number and shear parameter. Transition to non-stationary flow at $\beta \neq 0$ starts early than at $\beta=0$, in particularly flow becomes non-stationary if $\operatorname{Re}=280$ at $\beta=0.25$.

To investigate unsteady flow around sphere, small harmonic oscillations with frequency $\omega$ are imposed on inlet velocity $U=U_{a} \cos (\operatorname{Sh} t)$, where $U_{a}$ is amplitude of velocity oscillations, and $\mathrm{Sh}$ is the Strouhal number $\left(\mathrm{Sh}=\omega R / U_{a}\right)$. The flow is assumed to be axisymmetric. 


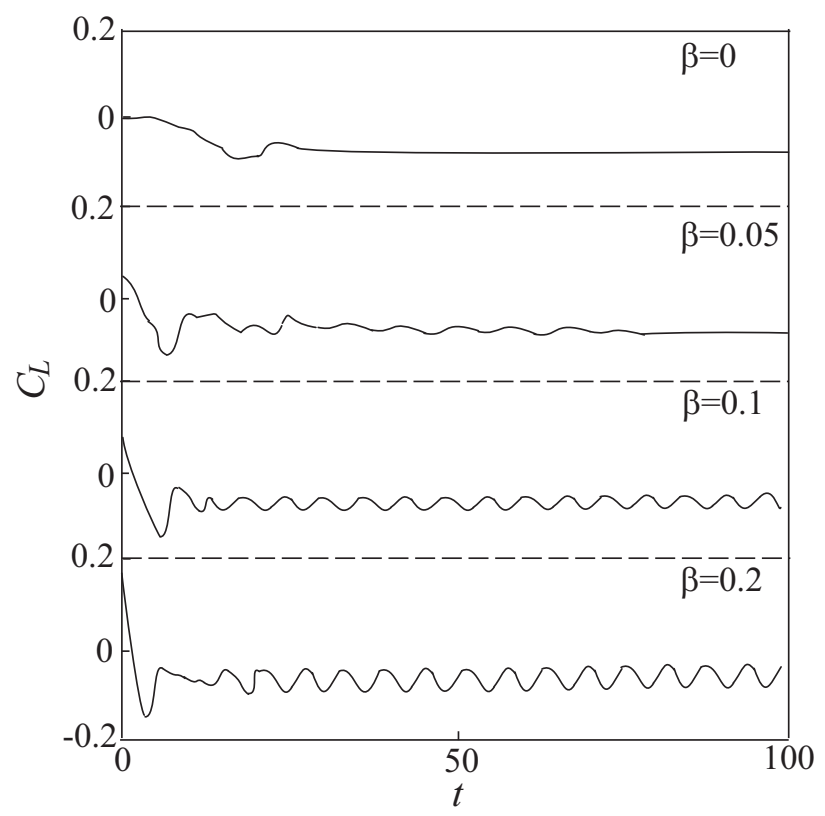

Figure 8. Time history of lift coefficient

Non-axisymmety of flow shows in the terms of order $\varepsilon^{2}$, where $\varepsilon$ is a ratio of amplitude of velocity oscillations to sphere radius [44]. Dependencies of friction coefficient and pressure coefficient on time are shown in the Figure 9 at $\mathrm{Sh}=\pi / 4$. The results calculated have qualitative agreement with the results of direct numerical simulation presented in $[45,46]$.
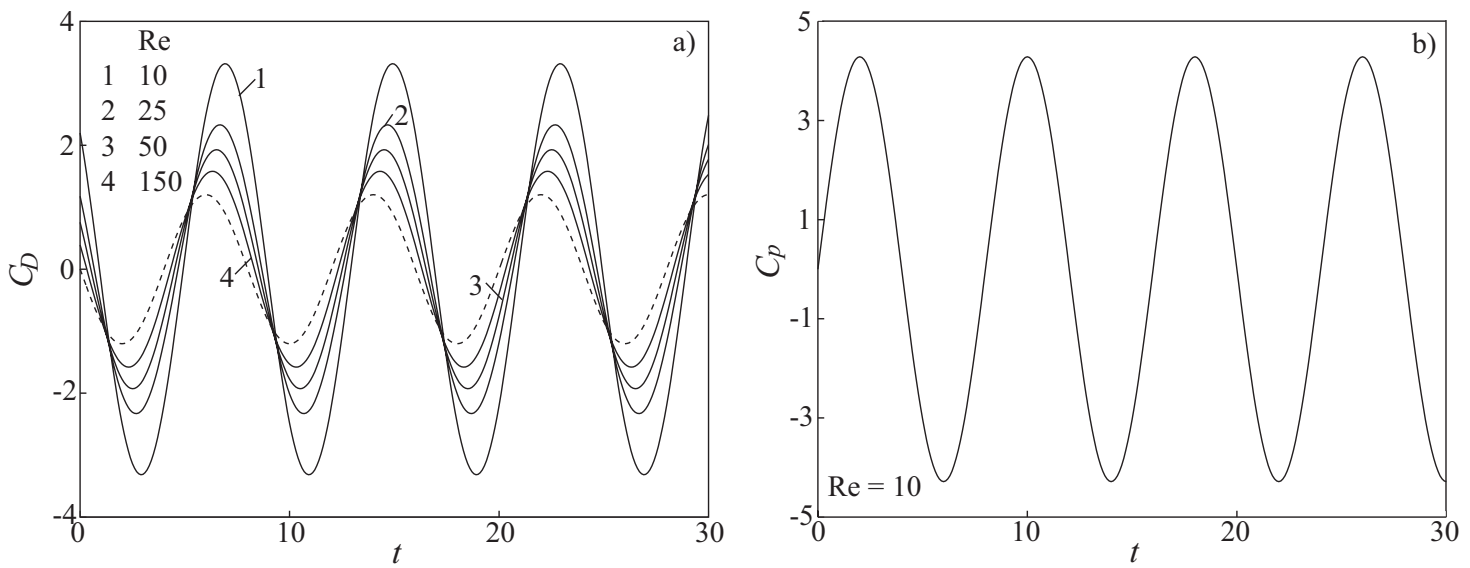

Figure 9. Time history of friction coefficient (a) and time history of pressure coefficient in stagnation point (b)

Inlet velocity changes the sign at $\varphi=\pi / 2$ and $3 \pi / 2$, where $\varphi=\operatorname{Sh} t$. The phase angle of drag coefficient depends on Reynolds number and Strouhal number, in particularly $\varphi=0.640$ at $\mathrm{Sh}=\pi / 4$, and $\varphi=0.584$ at $\mathrm{Sh}=\pi / 2$ (Reynolds number is fixed at 10 in both cases). Increase in Reynolds number at fixed Strouhal number leads to decrease in critical phase angle. Similar effect takes place at varied Strouhal number and fixed Reynolds number. The amplitude of drag coefficient oscillations is larger than the amplitude of velocity oscillations (dashed line in the Figure 9a). Influence of Reynolds number on friction coefficient is more considerable than its influence on pressure coefficient. This effect is related to the fact that pressure distribution along sphere at high Reynolds number $(\operatorname{Re}>150)$ is described with inviscid solution (except for sphere surface near the separation point). Pressure coefficient in stagnation point of sphere demonstrates weak sensitivity to the parameters of the flow, 
and is described by the inviscid solution.

\subsection{Combustion of droplet}

Radial temperature and species profiles are presented in the Figure 10. The scale corresponds to a dimensionless radius (the reference length is the droplet radius). Line 2 corresponds to aluminum, line 2 corresponds to aluminum oxide (AlO), line 3 and line 4 correspond to oxygen $\left(\mathrm{O}\right.$ and $\left.\mathrm{O}_{2}\right)$. It can be seen that the maximum of $\mathrm{AlO}$ specie appears at a distance of 2 droplet radii from the droplet surface.

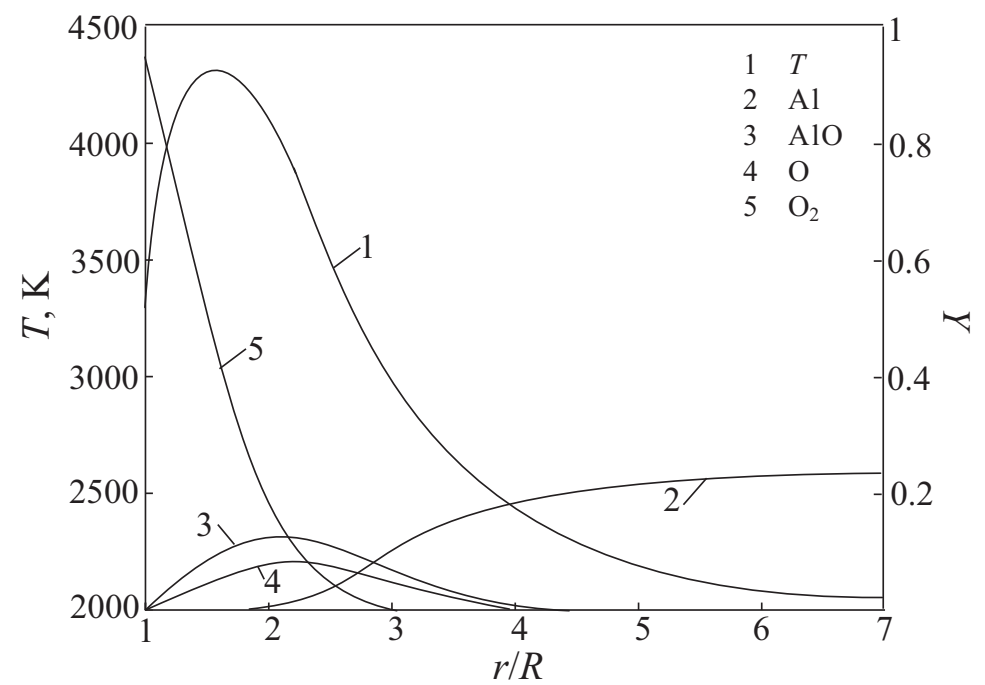

Figure 10. Profiles of temperature (line 1) and species (lines $2-5)$ at the front of the droplet

The particle heats up until its temperature reaches at the melting point of aluminum and the temperature is unchanged during the period of aluminum core melting because all the transferred heat is spent for supplying latent heat of fusion. As the particle temperature rises over $2200 \mathrm{~K}$, the oxide film is removed and the particle is ignited. Then the quasisteady combustion is initiated and the process transits from the ignition into diffusion controlled combustion regime.

The percentage of the droplet mass consumed is plotted against time from ignition out to approximately $120 \mathrm{~ms}$ in the Figure 11 . The mass consumed increases monotonically with time, with a constantly decreasing slope of the line. Approximately $60 \%$ of the mass has been consumed at $40 \mathrm{~ms}, 78 \%$ at $60 \mathrm{~ms}, 92 \%$ at $100 \mathrm{~ms}$, and $98 \%$ at $120 \mathrm{~ms}$ where the calculations are terminated.

In the Figure 12, the radius of aluminum droplet and the radius of the oxide cap are plotted against time. The radius of aluminum droplet decreases (slightly less than linearly) with time, while the radius of oxide cap increases with time, rapidly at first but at a decreasing rate with increasing time. Beyond approximately $42 \mathrm{~ms}$, the radius of oxide lobe is larger than the radius of droplet.

The relative amounts of aluminum and oxide are estimated as a part of the calculated burning time. Those results are shown in the Figure 13 for $100 \mu \mathrm{m}$ particle burning in a simulated propellant atmosphere at pressure of 1 atm $\left(T=2500 \mathrm{~K}, Y_{\mathrm{H}_{2} \mathrm{O}}=0.114, Y_{\mathrm{CO}_{2}}=\right.$ $\left.0.209, Y_{\mathrm{O}_{2}}=0.065\right)$. Two calculations are performed. One accounts for oxide accumulation on the particle, and the other neglects oxide formation. The calculation ignoring oxide formation gives a burning time the correlates with $D^{2}$-law, while the calculation allowing 


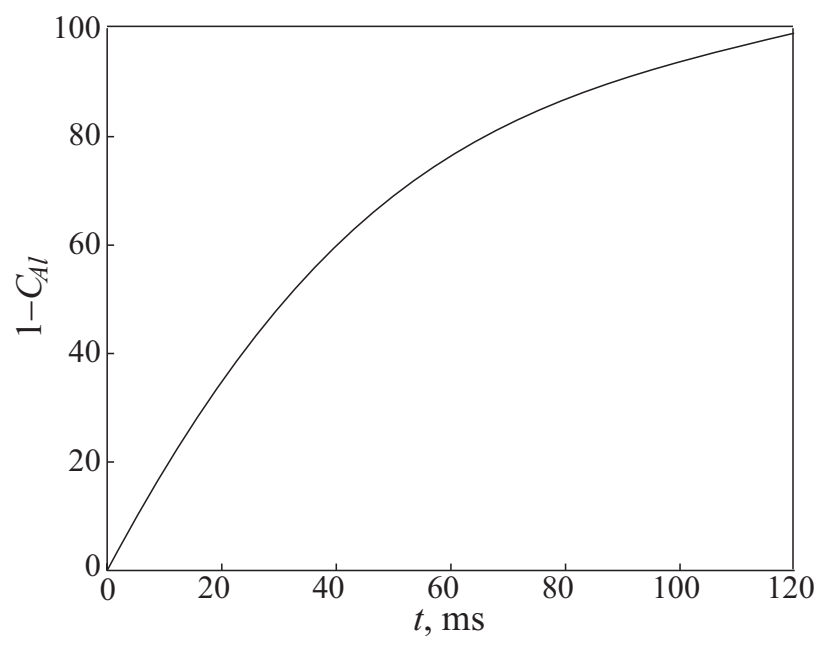

Figure 11. Time history of percentage aluminum burned

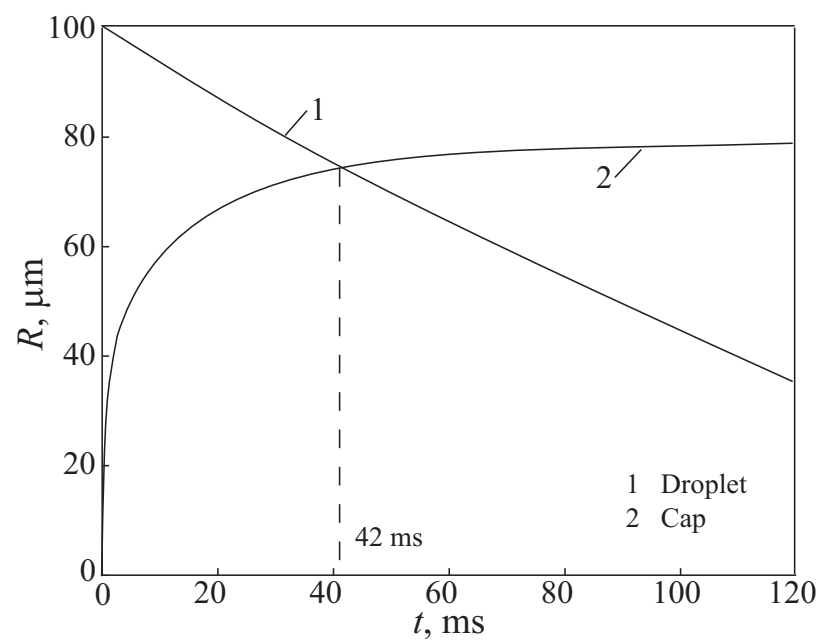

Figure 12. Time history of radius of aluminum droplet (line 1) and radius of oxide cap (line 2)

for oxide accumulation gives $D^{1.8}$ relationship. This value is in a good agreement with calculations based on the model of [38].

Since in classical diffusion-limited droplet combustion $D^{2}$-law applies with droplet radius squared decreasing linearly with time, the square of the predicted droplet radius is plotted against time in the Figure 13. Dashed line corresponds to the $D^{2}$-law, and solid line corresponds to the combustion of aluminium droplet with oxide cap. For the droplet with oxide cap, the analysis indicates that an exponential factor is about 1.8.

A significant deviation from $D^{2}$-behavior is observed with the absolute value of the slope of the line decreasing significantly with time. This behavior is largely a result of the fact that the fraction of the aluminum surface blocked by the oxide cap increases with time as shown in the Figure 14. Combustion efficiency is defined by the ratio of burning time to residence time of particles in the combustion chamber. In experimental investigations, the measured aluminum droplet combustion has varied from the simple $D^{2}$-model. A lower value of the exponent, varying from 2.0 to 1.2 , is observed in $[3,13,38]$. The statistical analysis, performed in [3], indicates that an exponential factor of 1.8 appears to correlate the data best.

Part of the aluminum oxide appears in the form of smoke at the condensation zone, while part deposits on an accumulating cap at the aluminum particle surface. The split is of 


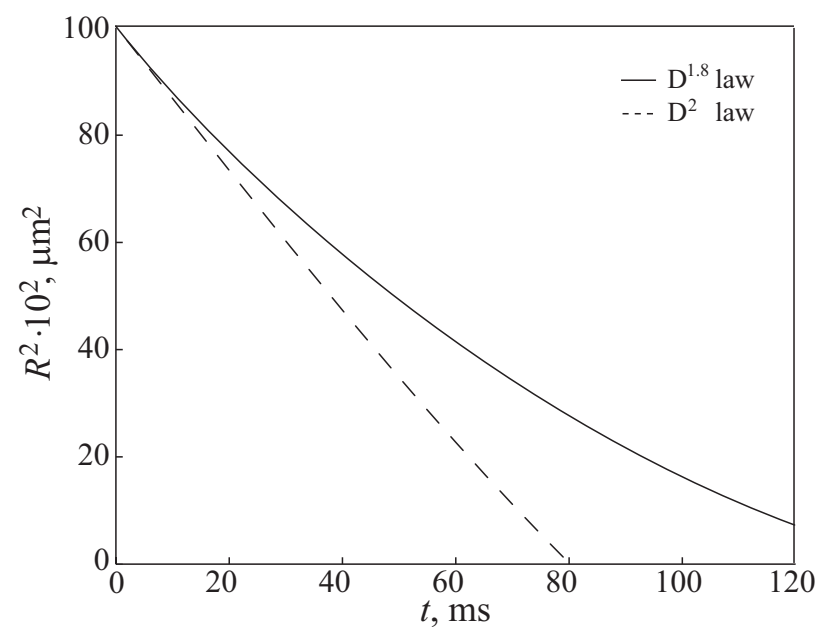

Figure 13. Time history of square of radius of aluminum droplet

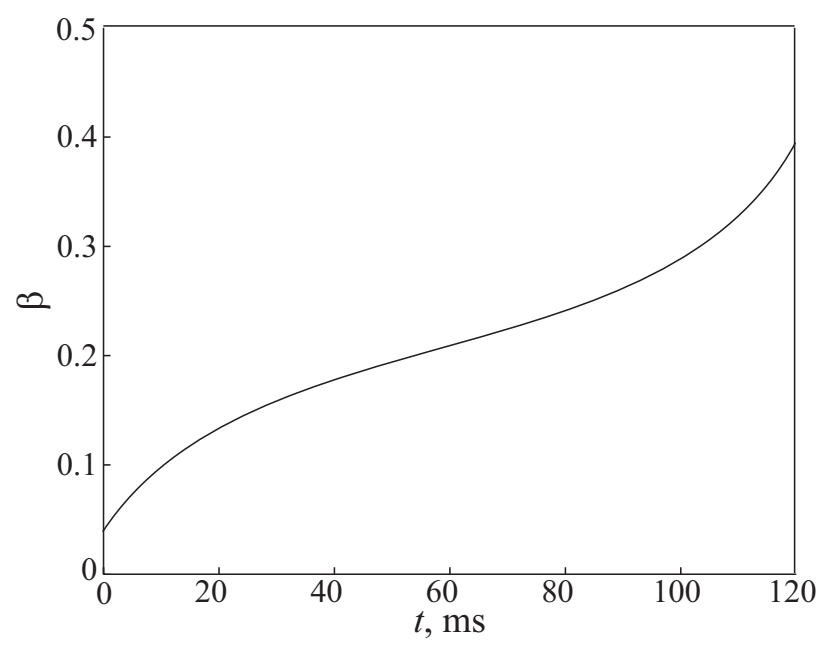

Figure 14. Time history of fraction of aluminum surface area blocked by oxide cap

interest due to different hydrodynamic behaviors of the two fractions. Accordingly, fraction of the oxide appearing in the cap is plotted against time in the Figure 15 which shows the asymptote to about $21 \%$.

In the Figure 16, sketches of the geometries of the aluminum and the oxide cap are presented on a common scale. The final oxide cap radius is predicted to be approximately $70 \%$ of the original aluminum droplet radius. A significant deviation from $D^{2}$-behavior is a result of the fact that the fraction of the aluminium surface blocked by the oxide cap increases with time.

The evaporation rate (slope of the $D^{2}$-line) of liquid aluminum droplet is distinguished from the decreasing rate of the particle surface area due to the inward diffusion and deposition of the combustion product to form a residue particle. The oxide cap grows due to the deposition of reaction product on the particle surface and remains as a residue particle after the completion of burning. The size of the residue particle is in the range from $1 / 2$ to $2 / 3$ of the initial particle diameter. 


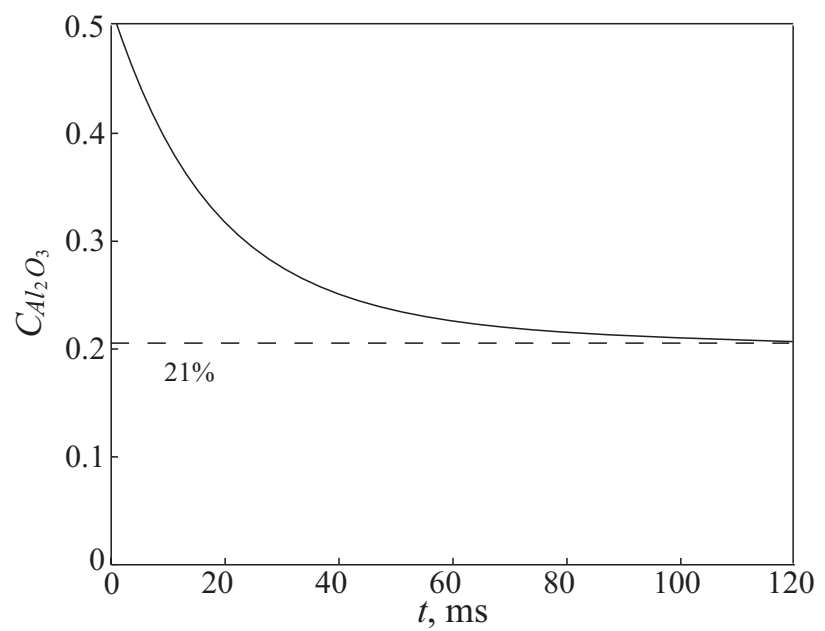

Figure 15. Time history of percentage of oxide product appearing in cap

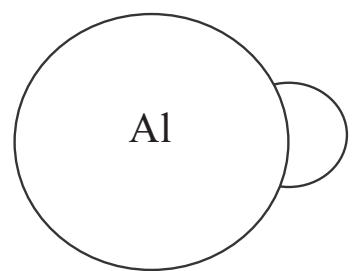

a)

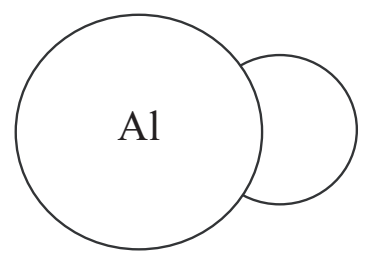

b)

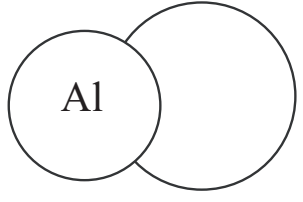

c)

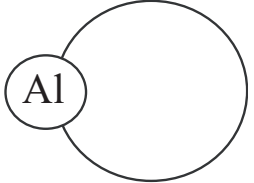

d)

Figure 16. Evolution of $\mathrm{Al}_{-} \mathrm{Al}_{2} \mathrm{O}_{3}$ shapes in time of $2 \mathrm{~ms}$ (a), $25 \mathrm{~ms}$ (b), $70 \mathrm{~ms}$ (c), and $120 \mathrm{~ms}(\mathrm{~d})$

\subsection{Burning time}

The combustion of aluminum particles is often characterized by the droplet burning time. Classical fuel droplet evaporation and combustion theory states the diameter of a burning droplet changes as $D^{2}=D_{0}^{2}-k t$, where $D$ is the particle diameter, $D_{0}$ is the initial particle diameter, $k$ is the burning rate (evaporation constant), and $t$ is the time. By setting droplet diameter to zero $(D=0)$, the burning time of droplet is defined as $t_{b}=D_{0}^{2} / k$. The evaporation constant is typically a function of the state properties of the medium including pressure, temperature and oxidizer concentration.

The combustion characteristics of individual aluminum droplet have been analyzed in [3], focusing on the burning time of individual particles. The wide variety of experimental techniques and a lack of standard definitions of the burning time, contribute to the large data scatter observed between different investigators. Past research has shown that the diameter dependence does not necessarily follow the classical $D^{2}$-law, but instead follows $D^{n}$-law, where $n$ is typically between 1.5 and 2 (lower values correspond to the small diameters and higher values correspond to the large diameters). It is assumed that $n$ is typically independent of the state properties of the medium, and is only a function of combustion mechanism. It has shown with classical relations that $n$ is 1.5 for laminar convective flows around the droplet and closer to 1 for turbulent flow [2]. The reason the exponent is lesser than 2 is due to the oxide cap and the convection, which causes the evaporation rate of aluminum to decrease and thus cause the burning time to increase. The oxide cap has another effect of providing energy to the particle surface during deposition, which cancels off some of the effect due to the blockage of the aluminum evaporation due to the oxide cap.

The particle burning time has to be approximated from the aluminum vaporization 
time. It is assumed that the steady-state vaporization rate of aluminum represented the initial vaporization rate and that thereafter the vaporization rate is proportional to the fraction of original aluminum remaining. In addition, when $95 \%$ of the original aluminum are vaporized, particle combustion is assumed to be completed. To evaluate the burning time from simulation results, the following expression is used

$$
t_{b}=\frac{1}{2} \rho_{A l} \int_{0}^{D_{0}} \frac{d D}{s_{A l}},
$$

where $\rho_{A l}$ is the density of liquid aluminum in the bulk phase and $s_{A L}$ is the mass consumption rate of bulk aluminum by surface reactions. The method consists in approximating the integral from a series of computational results on mass consumption rate for different diameters.

The burning time and local exponent found from calculations are presented in the Figure 17 (symbols $\square$ ). The burning time is also evaluated by correlation from [3] by taking $n=2$ (line 1 ) and $n=1.8$ (line 2). One can observe that the simulation results follow well these empirical trends. These results show an evolution from the diffusion-controlled regime with $n=2$ towards the combustion regime controlled by heterogeneous kinetics with $n=1$ when the droplet diameter decreases [28]. The differences are caused by several factors like the effect of oxidizer convection or the particularities of the transport model.

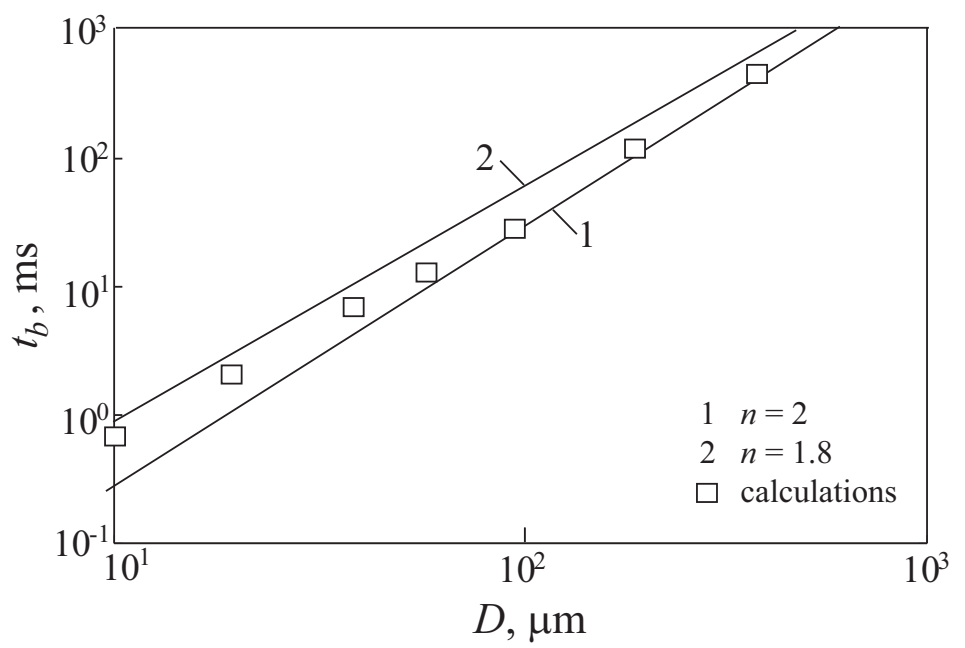

Figure 17. Burning time of the $D^{n}$ law versus droplet diameter

\section{Conclusion}

High-energy solid propellants are the mixtures of fuel, oxidizer and metal particles. Aluminum particles are introduced as an energetic component in solid propellant formulations to improve the thrust of SRMs. Metal particles burn in a gaseous atmosphere and produce condensed-phase residues. This process needs to be properly characterized in order to predict possible negative effects, for example thrust losses due to the dispersed phase. Aluminium droplet combustion and alumina residue behavior in the chamber affect combustion instabilities by acting as driving or damping mechanisms. 
Detailed modelling of individual particle combustion is a challenging problem because it needs to take into account complex physical and chemical phenomena. A numerical simulation of single aluminum droplet with oxide cap is performed. The burning time is evaluated for the considered physical conditions and modelling options. The results obtained highlight the crucial significance of the size and geometry of aluminium agglomerates on their burning rates. Detailed knowledge of condensed phase characteristics as residue size and burning time are essential to improve SRM performance and reliability.

\section{References}

[1] Price E.W. Combustion of metalized propellants. Progress in Aeronautics and Astronautics, 1984, 90, 479-513.

[2] Kuo K.K. Principles of combustion. New York, Wiley \& Sons, 1986.

[3] Beckstead M.W. A summary of aluminum combustion / VKI Special Course on Internal Aerodynamics in Solid Rocket Propulsion. Belgium, Rhode-Saint-Genese, 2002.

[4] Volkov K.N. Combustion of single aluminium droplet in two-phase flow / Heterogeneous Combustion. Nova Science, 2010, 191-260.

[5] Cohen N.S. A pocket model for aluminum agglomeration in composite propellants. AIAA Journal, 1983, 21(5), 720-725.

[6] Babuk V.A., Vasilyev V.A., Malakhov M.S. Condensed combustion products of the burning surface of aluminized solid propellant. Journal of Propulsion and Power, 1999, 15(6), 783-793.

[7] Maggi F., Bandera A., Galfetti L., DeLuca L.T., Jackson T.L. Efficient solid rocket propulsion for access to space. Acta Astronautica, 2010, 66(11-12), 1563-1573.

[8] Beckstead M.W., Liang Y., Pudduppakkam K.V. Numerical simulation of single aluminum particle combustion (review). Combustion, Explosion and Shock Waves, 2005, 41(6), 622-638.

[9] Dreizin E.L. Experimental study of stages in aluminum particle combustion in air. Combustion and Flame, 1996, 105(4), 541-556.

[10] Bucher P., Ernst L., Dryer F.L., Yetter R.A., Parr T.P., Hanson-Parr D.M. Detailed studies on the flame structure of aluminum particle combustion. Progress in Astronautics and Aeronautics, 2000, 689-722.

[11] Dreizin E.L. On the mechanism of asymmetric aluminum particle combustion. Combustion and Flame, 1999, 117(4), 841-850.

[12] Dreizin E.L. Phase changes in metal combustion. Progress in Energy and Combustion Science, 2000, 26(1), 57-78.

[13] Law C.K. A simplified theoretical model for the vapor-phase combustion of metal particles, Combustion Science and Technology, 1973, 7(5), 197-212.

[14] Gremyachkin V.M., Istratov A.G., Leipunskii O.I. Model for the combustion of metal droplets. Combustion, Explosion and Shock Waves, 1975 11(3), 313-318.

[15] Duterque J. Experimental studies of aluminum agglomeration in solid rocket motors. International Journal of Energetic Materials and Chemical Propulsion, 1997, 4(1-6), 693-705. 
[16] Widener J.F., Liang Y., Beckstead M.W. Aluminum combustion modeling in solid propellant environments. AIAA Paper, 99-2629.

[17] Liang Y., Beckstead M.W. Numerical simulation of quasi-steady, single aluminium particle combustion in air. AIAA Paper, 98-0254.

[18] Malinin V.I., Kolomin E.I., Antipin I.S. Combustion of aluminum particles in flows of reactive gases. Combustion, Explosion and Shock Waves, 1999, 35(1), 36-42.

[19] Babuk V.A., Vasilyev V.A. Model of aluminum agglomerate evolution in combustion products of solid rocket propellant. Journal of Propulsion and Power, 2002, 18(4), 814823.

[20] Trunov M.A., Schoenitz M., Zhu X., Dreizin E.L. Effect of polymorphic phase transformations in $\mathrm{Al}_{2} \mathrm{O}_{3}$ film on oxidation kinetics of aluminum powders. Combustion and Flame, 2005, 140(4), 310-318.

[21] Dreizin W.L., Trunov M.A. Surface phenomena in aluminum combustion. Combustion and Flame, 1995, 101(3), 378-382.

[22] DesJardin P.E., Felske J.D., Carrara M.D. Mechanistic model for aluminum particle ignition and combustion in air. Journal of Propulsion and Power, 2005, 21(3), 478-485.

[23] King M.K. Aluminum combustion in solid rocket motor environment. Proceedings of the Combustion Institute, 2009, 32(2), 2107-2114.

[24] Hermsen R.W. Aluminum oxide particle size for solid rocket motor performance prediction. Journal of Spacecraft Rockets, 1981, 18(6), 483-490.

[25] Gan Y., Qiao L. Combustion characteristics of fuel droplets with addition of nano- and micron-sized aluminum particles. Combustion and Flame, 2011, 158, 354-368.

[26] Muller M., Davidenko D., Giovangigli V. Computational study of aluminum droplet combustion in different atmospheres. Proceedings of the 7th European Conference for Aeronautics and Space Sciences (EUCASS), 3-6 July 2017, Milan, Italy, 17 p.

[27] Yang J., Yoon W. Modeling of aluminum particle combustion with emphasis on the oxide effects and variable transport properties. Journal of Mechanical Science and Technology, 2010, 24(4), 909-921.

[28] Glorian J., Gallier S., Catoire L. On the role of heterogeneous reactions in aluminum combustion. Combustion and Flame, 2016, 168, 378-392.

[29] Gross M.L., Felt S.A., Beckstead M.W. Two-dimensional modeling of AP composite propellants with detailed kinetics: particle size effects. AIAA Paper, 2006-4925.

[30] Rashkovskii S.A. Role of the structure of heterogeneous condensed mixtures in the formation of agglomerates. Combustion, Explosion and Shock Waves, 2002, 38(4), 435445.

[31] Wang X., Jackson T.L., Buckmaster J. Numerical simulation of the three-dimensional combustion of aluminized heterogeneous propellants. Proceedings of the Combustion Institute, 2007, 31(2), 2055-2062.

[32] Betelin V.B., Smirnov N.N., Nikitin V.F., Dushin V.R., Kushnirenko A.G., Nerchenko V.A., Evaporation and ignition of droplets in combustion chambers modeling and simulation. Acta Astronautica, 2012, 70, 23-35.

[33] Smirnov N.N., Betelin V.B., Kushnirenko A.G., Nikitin V.F., Dushin V.R., Nerchenko V.A. Ignition of fuel sprays by shock wave mathematical modeling and numerical simulation. Acta Astronautica, 2013, 87, 14-29. 
[34] Smirnov N.N., Nikitin V.F., Dushin V.R., Filippov Yu.G., Nerchenko V.A., Khadem J. Combustion onset in non-uniform dispersed mixtures. Acta Astronautica, 2015, 115, 94-101.

[35] Emelyanov V.N., Teterina I.V., Volkov K.N., Garkushev A.U. Pressure oscillations and instability of working processes in the combustion chambers of solid rocket motors. Acta Astronautica, 2017, 135, 161-171.

[36] Tyurenkova V.V. Non-equilibrium diffusion combustion of a fuel droplet. Acta Astronautica, 2012, 75, 78-84.

[37] Dreizin E.L. Experimental study of aluminum particle flame evolution in normal and micro-gravity. Combustion and Flame, 1999, 116(3), 323-333.

[38] Brooks K.P., Beckstead M.W. Dynamics of aluminum combustion, Journal of Propulsion and Power, 1995, 11(4), 769-780.

[39] Guillen D.P., Williams B.G. In-flight oxidation of aluminum in the twin-wire electric arc process. Journal of Thermal Spray Technology, 2006, 15(1), 63-71.

[40] Roberts T. Ignition and combustion of aluminum/magnesium alloy particles in $\mathrm{O}_{2}$ at high pressures. Combustion and Flame, 1993, 92(1), 125-143.

[41] Volkov K. Multigrid and preconditioning techniques in CFD applications / CFD Techniques and Thermo-Mechanics Applications. Springer International Publishing, 2018, 83-149.

[42] Smirnov N.N., Betelin V.B., Nikitin V.F., Stamov L.I., Altoukhov D.I. Accumulation of errors in numerical simulations of chemically reacting gas dynamics. Acta Astronautica, 2015, 117(1), 338-355.

[43] Olsen S.E., Beckstead M.W. Burn time measurements of single aluminum particles in steam and carbon dioxide mixtures. Journal of Propulsion and Power, 1996, 12(4), $662-671$.

[44] Higa M., Takahashi T. Stationary flow induced by an unharmonically oscillating sphere. Journal of the Physical Society of Japan, 1987, 56(5), 1703-1712.

[45] Chang E.J., Maxey M.R. Unsteady flow about a sphere at low to moderate Reynolds number: oscillatory motion. Journal of Fluid Mechanics, 1994, 277, 347-379.

[46] Chang E.J., Maxey M.R. Unsteady flow about a sphere at low to moderate Reynolds number: accelerated motion. Journal of Fluid Mechanics, 1995, 303, 133-153. 